\title{
Informationsgewinnung im cyberphysischen Produktionssystem
}

\author{
René von Lipinski*, Markus Richter, Jörg Reiff-Stephan
}

\section{Zusammenfassung}

Im Fokus vieler industrieller Forschungsprojekte steht die intelligente Vernetzung von Entitäten im produktionstechnischen Umfeld. Im Rahmenkonzept Industrie 4.0 wird zum Wandel bestehender hierarchischer Betriebsstrukturen in ein cyberphysisches Produktionssystem beigetragen. Priorität haben Untersuchungen sensorischer Komponenten und deren Verkettung als Führungselement im zukünftigen Produktionsumfeld, welches durch Selbstanalyse, Selbstorganisation und Selbstoptimierung geprägt sein wird. Ziel des Hochschulprojektes (iSensPK) ist die Abwandlung bestehender Sensorkonzepte und deren Nutzbarmachung für das Internet der Dinge. Einen Schwerpunkt wird dabei insbesondere die Definition und Verknüpfung stabiler Kommunikationswege darstellen. Als Demonstrator erfolgt der Aufbau eines 6-Komponenten Kraftmesssystems zur Prüfung und Kalibrierung moderner Greifertechnologien unter Feldbedingungen. Durch die umfangreiche Vernetzung verschiedener Sensoren erfolgt die Erfassung von Störgrößen im Testumfeld. Das gewonnene Abbild der physischen Umgebung dient zur Korrektur der Kraftmessungsergebnisse. Für die Realisierung eines derartigen Kraftmess- und Umfelderfassungssystems wird in den sensorisch relevanten Bereichen der Kommunikation, Sensordatenfusion, Multifunktionsintegration und Selbstorganisation nach Lösungsansätzen gesucht.

\section{Abstract}

The main focus of different industrial R\&D projects explores possible starting points for the intelligent interconnection of entities in a productional environment. Against a background of 'Industrie 4.0', this contributes to a transformation of existing, hierarchical structures into a cyber physical production system. The focus here is on investigating sensor components and their interlinking as a main element in a future production environment which will be characterised by self-analysis, self-organisation and self-optimisation. The aim of the project iSensPK is to modify sensor concepts in being to facilitate their utilisation in the Internet of Things. Emphasis rests on defining and combining stable lines of communication. A demonstrator is set up, including a six-component force measuring system to enable inspecting and calibration of modern gripping technologies within field conditions. Through adequate linkage of different sensors, the capture of disturbance variables is made possible. The resulting image of the physical environments serves to readjust any measured force values. To implement such a force measuring and environment detecting system, sensorial relevant fields of communication, sensor data fusion, multifunctional integration and self-organisation are searched to conclude approaches to solutions.

\section{EINLEITUNG}

Wirtschaftliche Produktionsprozesse sind in zunehmendem Maße unter dem Blickwinkel der Effektivität und Effizienz auf dem globalen Anbietermarkt zu steuern. Hierbei wird die Aufnahme und Verarbeitung von Informationen als einer der wichtigste Innovationstreiber in der industrialisierten Produktionswelt angesehen. Das Internet der Dinge/ Dienstleistungen/Daten (iD3) manifestiert sich hierbei in dem Szenario der ,Industrie 4.0“- Produktions-Gesellschaft.
Der Begriff Industrie 4.0 (kurz: i4.0) steht für die vierte industrielle Revolution (Abbildung 1) und ist ein Teilaspekt der Hightech-Strategie der Deutschen Bundesregierung (Kagermann et al. 2013). Durch vertikale und horizontale Vernetzung industrieller Strukturen entstehen neue Wertschöpfungsnetzwerke, welche auch zukünftig erfolgreich am weltweiten Markt partizipieren sollen.

Was die vierte industrielle Revolution jedoch deutlich von vorhergegangenen unterscheidet, ist die Tatsache, dass sie nicht auf einzelnen, bahnbrechenden technologischen Neuerungen beruht. Vielmehr ist Industrie 4.0 geprägt von der Symbiose bestehender Technologien. Im Fokus stehen hier unter anderem Anwendungen aus den Bereichen der Informatik, Kommunikationstechnik, Aktorik und Sensorik. Der freie Zugang zu Informationen und eine umfassende Vernetzung gehören heute im Privatleben vieler Menschen zum Alltag. Mit Hochdruck wird an Konzepten zur Übertragung derartiger 


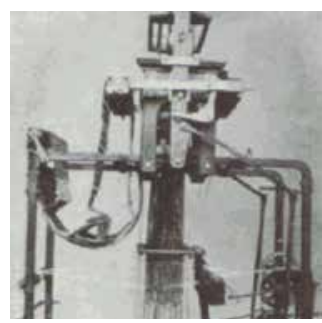

1787 Erster mechanischer Webstuhl

1. Industrielle Revolution

durch Einführung mechanischer Produktionsanlagen (Dampfkraft)

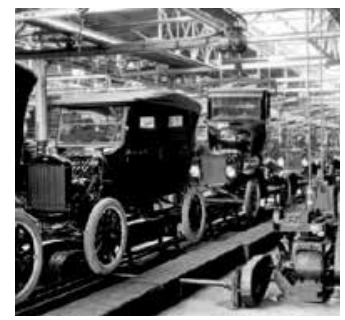

1870 Erstes Fließband, Schlachthöfe von Cincinnati

\section{Industrielle Revolution}

durch Einführung arbeitsteiliger Massenproduktion (Elektrische Energie)

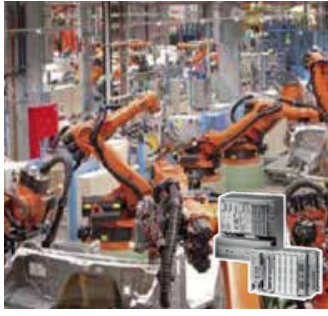

1969 Erstes SPS

Allen Bradley

Modicon 084

3. Industrielle Revolution

durch Einsatz von Elek-

tronik und Informations-

technik zur weiteren

Automatisierung der

Produktion

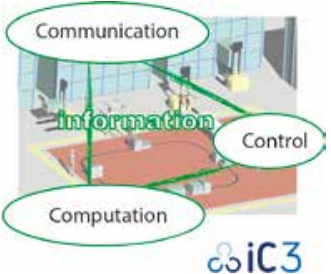

2011 Smart Factory Internet of Things

4. Industrielle Revolution

auf Basis und unter

Nutzung von cyber-

physischen Systemen (CPS)

\begin{tabular}{llll}
\hline Ende des & $\vdots$ & & \\
18. Jahrhunderts & Beginn des & Beginn der 70er Jahre & Zeit \\
20. Jahrhunderts & des 20. Jahrhunderts
\end{tabular}

Abb. 1) Übersicht der vier industriellen Revolutionen.

Strukturen auf das industrielle Umfeld geforscht. Ziel der Forschung ist die Realisierung des Internets der Dinge sowie cyberphysischer Produktionssysteme (CPPS). Diese sollen zu einem Paradigmenwechsel innerhalb der deutschen Industrielandschaft führen und die hierarchischen Strukturen der gegenwärtigen Automatisierungspyramide ablösen (Abbildung 2) (Spath 2013, Sauerer 2013).

CPPS bestehen aus einer Summe eingebetteter Systeme, welche ihre Umwelt sensorisch erfassen und durch Aktoren auf selbige einzuwirken vermögen (Zhang et al. 2013, Reinhart et al. 2013).

Durch Verbindung mit digitalen Netzen können verfügbare Daten und Dienste genutzt werden, zudem ist eine Schnittstelle zur Mensch-Maschine-Kommunikation vorgesehen. Ein wichtiger Punkt ist hierbei unter anderem die Fähigkeit zum Sammeln, Auswerten und Bereitstellen von Daten (Vogel-Heuser et al. 2013). Vielfältig existieren bereits technische Lösungen zur Umsetzung der Industrie 4.0-Ansätze in bestehende oder neue Produktionsketten. Es gilt nun, aus der Vielfalt von Sensorik-, Aktorik- und Netzwerkkomponenten auszuwählen und aus diesen sinnvoll ein Gesamtsystem zusammenzustellen.

Im Rahmen des Projektes „Intelligente Sensorsysteme für selbstoptimierende Produktionsketten" (iSensPK) wird an der Nutzbarmachung bestehender Sensortechnologie für cyberphysische Produktionssysteme geforscht. Eine Prüfeinrichtung für die Kraftmessung an unterschiedlichen Greifersystemen dient hierbei als Demonstrator.
Durch die Vernetzung von Sensoren soll ein intelligentes Umfelderkennungssystem geschaffen werden. Das genaue Abbild der Umgebungsbedingungen soll dabei helfen, die Ergebnisse der Kraftmessungen zu verbessern. Zusätzlich werden die Testbedingungen und Ergebnisse protokolliert und im digitalen Gedächtnis des Greifers festgehalten. Diese Daten können dann von anderen Entitäten im Produktionsprozess abgefragt werden. Erfüllt ein Greifer nicht die für den Handhabungsprozess notwendigen Parameter (ReiffStephan 2013), kann dann die entspre-

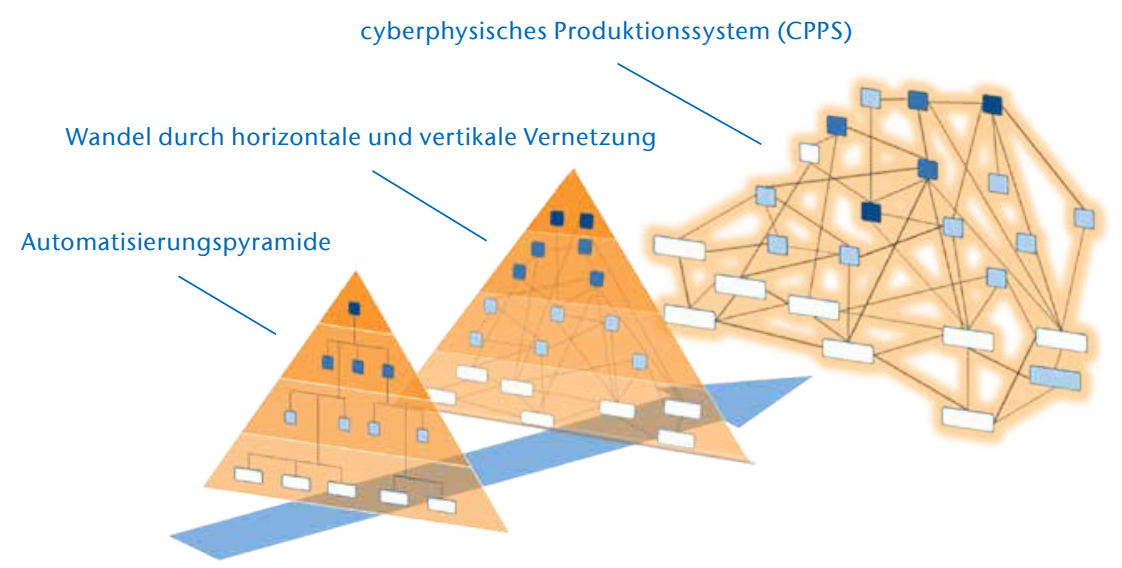

Abb. 2) Wandel der Automatisierungspyramide zum cyberphysischen Produktionssystem 
chende Entität den Einsatz des Greifers verweigern und somit unsichere Situationen vermeiden.

\section{MARKTANALYSE I4.0-FÄHIGER KOMPONENTEN}

\subsection{KLASSIFIKATIONSANSATZ}

Richtlinien und Normen zur Beurteilung, ob eine technische Lösung den Anforderungen einer Industrie-4.0Anwendung gerecht wird, befinden sich im Entstehungsprozess. Ein erster Ansatz für eine entsprechende Klassifizierung resultiert aus den Bemühungen des VDI. Ein Bewertungsschema, ähnlich dem der von Elektrogeräten bekannten IP-Schutzklassen, beurteilt ein System oder einzelne Komponenten nach deren Kommunikationsfähigkeit und deren Bekanntheitsgrad im Informationssystem (siehe Abbildung 3) (Epple et al. 2014).

Technische Lösungen mit einem Communication-Presentation Index (CP) von CP43 oder CP44 werden demnach als 14.0-Komponenten bezeichnet. 14.0Komponenten können einzelne Bauteile, aber auch Subsysteme sein, deren Bestandteile erst als Baugruppe die geforderte CP-Klasse erfüllen (Epple et al. 2014). Im Folgenden soll näher auf die am Markt erhältlichen Lösungen aus dem Bereich der Sensorik eingegangen werden, welche sich für die Realisierung einer 14.0-Komponente eignen.

\subsection{INTELLIGENTE SENSOREN}

Die neueste, auf dem Markt vertretene Generation intelligenter Sensoren wird auch als Smart Sensor bezeichnet. Dabei gibt es einige Anforderungen, die eine solche Komponente für den erfolgreichen Einsatz in einer Industrie 4.0-Umgebung erfüllen muss. Bei Anschluss eines Sensors muss sich dieser selbstständig im System anmelden und eine eindeutige Identifizierung ermöglichen. Durch Selbstdiagnose sind die Sensoren in der Lage, Störungen und Einbrüche der Performance zu erkennen und selbstständig anzuzeigen (Reiff-Stephan \& Lipinski 2014).

Unterstützt durch die Lokalisierung des Sensors am Mensch-MaschineInterface ist so eine zeitnahe War-

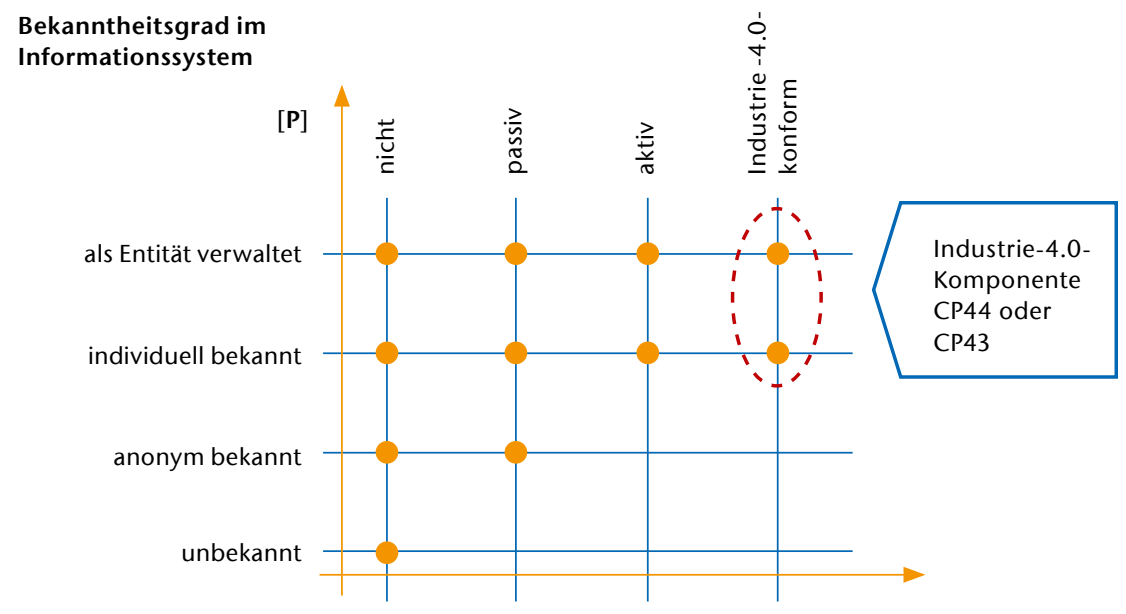

Kommunikationsfähigkeit [C]

Abb. 3) Klassifizierungsansatz 14.0-Komponenten nach Epple et al. (2014).

tung möglich. Dank Speicherung der applikationsspezifischen Parameter der Sensoren kann im Versagensfall ein 1:1-Austausch erfolgen, da das Automatisierungssystem automatisch die Richtigkeit des neuen Sensors prüft und die Parameter setzt. Gleicherweise können auch für verschiedene Produktionsaufgaben die entsprechenden Einstellungen hinterlegt und bei Bedarf eingespielt werden. Ein wesentlicher Aspekt liegt jedoch in der Dezentralisierung intelligenter Funktionen, sodass Sensoren immer mehr Anteil an der Steuerung nehmen, etwa lediglich für den Regelungsprozess relevante Daten weitergeben, beispielsweise erst dann, wenn Abweichungen vom Normal auftreten. Diese Vorgehensweise stellt sicher, dass weder Bandbreite noch Rechenkapazität der Steuerung mit überflüssigen Informationen belastet werden, die den Prozess stören. Vorteilhaft ist auch, dass nicht erst auf eine Verarbeitung und Rückmeldung des zentralen Steuersystems gewartet werden muss, sodass die für Hochgeschwindigkeitsanwendungen notwendigen, minimalen Reaktionszeiten erreicht werden können. Selbst auf die Verwendung statistischer Daten zum Abgleich muss nicht verzichtet werden, da diese auf den Sensoren hinterlegt werden können. Die Steuerung muss dann im Optimalfall lediglich ein binäres Signal auswerten (Sauerer 2013).

\subsection{KOMMUNIKATIONS- TECHNOLOGIEN}

Für die Kommunikation zwischen Sensoren, Aktoren und Steuerungseinheiten gibt es verschiedene Schnittstellen und Kommunikationswege. Durch den vermehrten Einsatz von Sensoren wird es zunehmend wichtiger, ein zuverlässiges, kollisionsfreies Netzwerk aufzubauen. In der Feldebene werden Sensoren und Aktoren üblicherweise mit einem Bussystem verbunden. Nachteilig ist hier die Inkompatibilität der verschiedenen Systeme zueinander. Unterschiede bestehen in physikalischen Spezifikationen und verschiedenen Infrastrukturkomponenten. Selbst Sensoren sind häufig bereits für ein bestimmtes Bussystem ausgelegt, zusätzlich hat jeder Sensorhersteller seine eigene Schnittstelle entwickelt. Die aus Variantenvielfalt und Kompatibilitätsproblemen resultierenden Nachteile führen zu zwei Standardisierungstrends im Bereich der Sensorik. Einerseits die Verwendung einer universellen Schnittstelle, wobei sich IO-Link vielversprechend zeigt. Andererseits die Verwendung einer standardisierten Kommunikationstechnologie, wobei sich die bereits bestehende Struktur des Ethernets anbietet. So wird auch der Einsatz diverser Funktechnologien erleichtert, wobei sowohl W-LAN als auch Bluetooth in ihren Varianten Anwendung finden. 
Das bislang in der Sensorik verwendete Interface nach DIN EN 60947-5-2 ist für die heutigen Sensoranwendungen schon lange nicht mehr ausreichend, da sich nur ein oder zwei Schaltbits übertragen lassen. Viele Hersteller haben deshalb eigene, spezielle Schnittstellen entwickelt, sodass auf dem Markt vielfältig Multipolstecker und -kabel zu finden sind, je nachdem welcher Sensor damit integriert werden soll (Kräußlich 2013). Der „USBStandard für die Fabrikautomation" wie der IO-Link auch genannt wird, setzt genau an dieser Stelle an. Den Anstoß dazu gab Siemens durch Herantreten an die Sensorhersteller und die resultierende Gründung des IOLink-Konsortiums, im Rahmen dessen man sich auf ein Interface mit rückwärtskompatibler Dreileiter-Physik einigte. Somit ist es möglich, sowohl herkömmliche als auch hochkomplexe Sensoren parallel in einem System zu betreiben und dabei für die digitale IO-Link-Übertragung lediglich ein ungeschirmtes, dreiadriges Industriekabel zu verwenden (Abbildung 4). Dadurch sind sämtliche Sensoren und Aktoren unabhängig von den in der Steuerungsebene verwendeten Bussystemen, da sie lediglich via Punktzu-Punkt-Kommunikation mit dem IOLink-Master verbunden sind. Weitere Vorteile liegen in den Gewichts- und Materialeinsparungen sowie der geringeren Kabelbruchgefahr im Industriekabel.

Der in der IEC 61131-9 definierte Standard ermöglicht somit eine wenig komplexe, bidirektionale Kommunikation, über welche etwa Parameter gesetzt und auf später oder ersatzweise angeschlossene, baugleiche Sensoren übertragen werden können. Zudem ist jede IO-Link-Komponente über die IODD, die IO-Link Device Description, eindeutig identifizierbar und in der Lage, sich eigenständig mit einer vollen Funktionsbeschreibung in einem System anzumelden - gemeinsam mit der Kommunikationsfähigkeit eine der Grundvoraussetzungen für das zukünftige Industrie-4.0-Sensorkonzept.
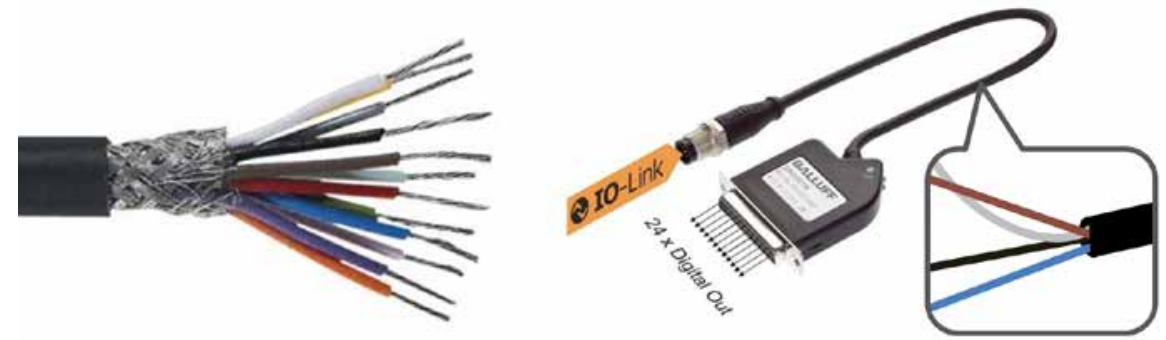

Abb. 4) Verglichen mit konventionellen Kabeln (links) benötigen IO-Linkkabel weniger Adern für die Realisierung mehrerer digitaler Ausgänge (rechts) (CNC-kompakt n/a., SPS-MAGAZIN 2011).

\subsection{INDUSTRIELLES ETHERNET}

Das offene Ethernet-Protokoll ermöglicht eine direkte, vertikale Integration, wobei sich das industrielle Ethernet jedoch vom Standard nach IEEE 802.3, wie er üblicherweise in Büronetzwerken Verwendung findet, abhebt. Weltweit kommen rund 30 verschiedene Industrial-Ethernet-Systeme zum Einsatz, häufig haben dabei FeldbusHersteller eigene Protokolle geschrieben. Zu den bekanntesten Systemen zählen etwa PROFINET, POWERLINK, EtherNet/IP, EtherCat und SERCOS III. Entscheidend sind die Unterschiede zum Standard-Ethernet, insbesondere betreffs der Echtzeitfähigkeit, d.h. der Fähigkeit, sämtliche Datenpakete innerhalb eines vorgegebenen Zeitrahmens (harte Echtzeit) oder mit einer noch zulässigen Abweichung (weiche Echtzeit) zu übermitteln. Dabei bewegen sich die zu erreichenden Zykluszeiten bzw. Taktungenauigkeiten (Jitter) im zwei- bis dreistelligen Millisekundenbereich - etwa bei Temperaturmessungen - bis hin zu Mikrosekunden für digitale Regelsysteme oder Motion-Control-Anwendungen, siehe auch (Meindl 2014). Die Vorzüge des Ethernets liegen insbesondere in den bereits genormten Steckerverbindungen sowie in der im Vergleich zu Bussystemen unweit größeren verfügbaren Bandbreite. So lassen sich beispielsweise mit einem PROFIBUSSystem (Siemens) $12 \mathrm{MBit} / \mathrm{s}$ erreichen, während sich Ethernet bereits in Bereichen jenseits der $1 \mathrm{GBit} / \mathrm{s}$-Grenze bewegt (Lachello et al. 2013).

\section{TESTSTAND-VERSUCHSAUFBAU}

Resultierend aus den getroffenen Vorüberlegungen werden verschiedene Sensoren für den Einsatz im Teststand ausgewählt. Als Fallbeispiel dient hierbei eine sechsachsige Kraftmessung für verschiedene Greifersysteme bei gleichzeitiger Erfassung der Umgebungsparameter. Berücksichtigt werden unter anderen Druck, Temperatur und Feuchtigkeit der Umgebungsluft, aber auch Drücke innerhalb pneumatischer Greiferkomponenten oder die Werkstücktemperatur beim Einsatz von Gefriergreifern. Durch Abgleich und Auswertung der Daten soll die Qualität der Kraftmessung erhöht sowie realisiert werden. Zudem kommen verschiedene Kommunikationsschnittstellen zum Einsatz, So werden die Austauschbarkeit von Komponenten überprüft sowie Vor- und Nachteile abgebildet. Abbildung 5 zeigt ein digitales Mock-Up des geplanten Aufbaus. Kernstück des Teststands bildet eine einachsige Lineareinheit, welche eine Kraft von bis zu $1.000 \mathrm{~N}$ in das System einbringen kann. Durch exzentrischen Kraftangriff können auch mehrachsige Spannungszustände hervorgerufen und betrachtet werden. Der Teststand ist mobil ausgeführt, sodass er in verschiedenen Umgebungen mit entsprechend variierenden Umgebungsbedingungen zum Einsatz gebracht werden kann. Für die Kraftmessung stehen verschiedene Greifersysteme zur Auswahl. Neben einem gewöhnlichen Zwei-Finger-Greifer können auch Gefriergreifer für biegeschlaffe Bauteile getestet werden. Diese benetzen das 


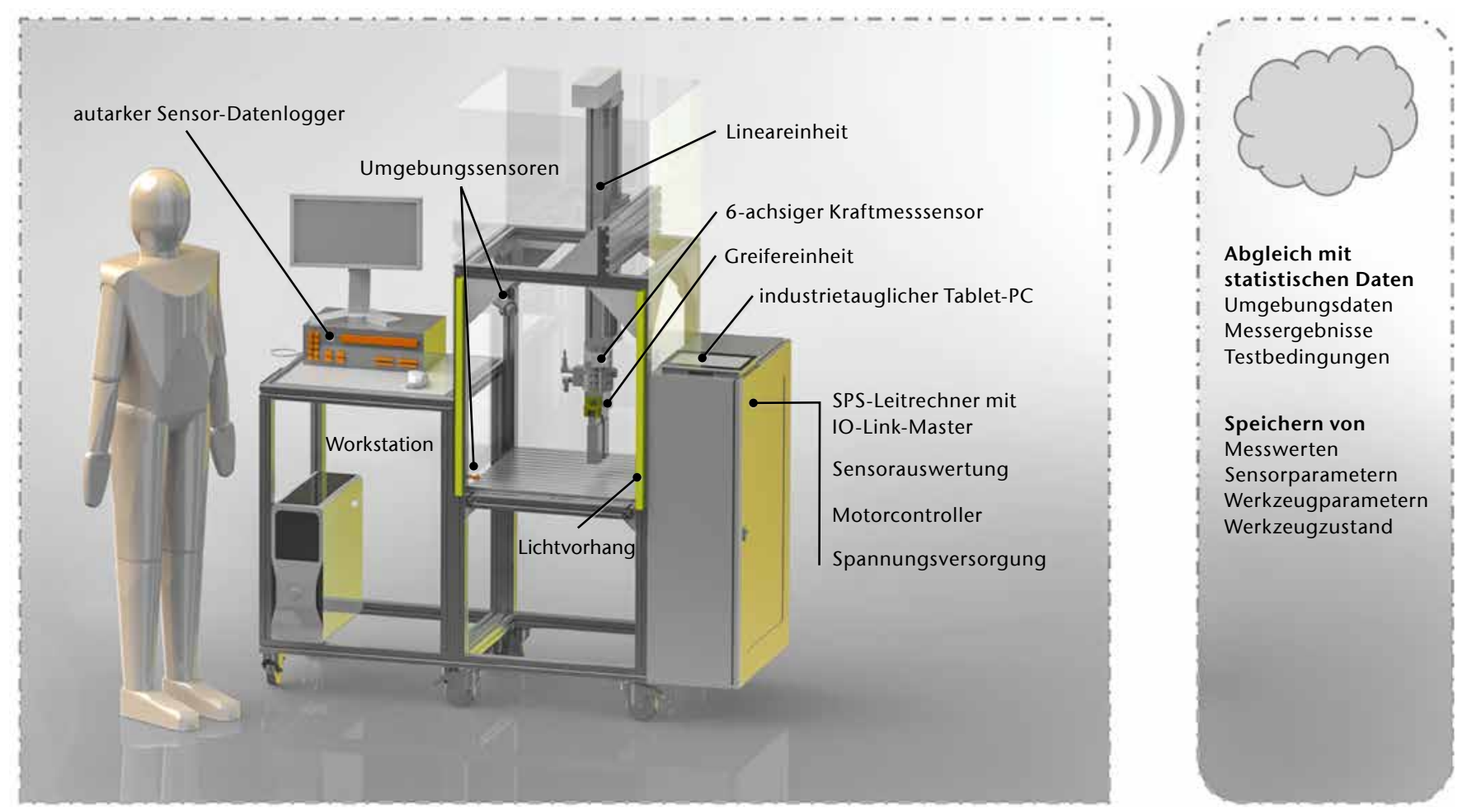

Abb. 5) 3D-Modell des Teststands mit Erläuterung.

Bauteil mit einem dünnen Wasserfilm und schaffen durch Unterschreiten dessen Gefrierpunkts eine hydroadhäsive Verbindung. Je nach Positionierung des Kraftmesssensors auf der Grundplatte oder direkt an der Greifereinheit können unterschiedliche Feldbedingungen wie etwa der Abriss eines Bauteils simuliert werden. Im weiterführenden Einsatz ist es denkbar, das Linearsystem mit einer Robotereinheit zu substituieren und so auch dynamischere Messungen, beispielsweise für auftretende Beschleunigungen bei Schwenkbewegungen, durchzuführen.

Zentrale Komponente des Kraftmesssystems ist ein sechsachsiger, auf Silizium-Dehnmessstreifen basierter Kraftmesssensor. Die Auswahl eines entsprechenden Sensors erfolgt auf Basis einer Marktanalyse bezüglich Netzwerktauglichkeit und Auflösung. Deren Ergebnisse zeigen, dass die eigenständige Netzwerkanmeldung und Eingliederung des Sensors über Ethernet-Schnittstelle und MAC-Adresse in Kombination mit einer sehrfeinen Auflösung von 1,0 - 1,5\% (je nach Achse) momentan nur durch einen Kraftmesssensor des Herstellers Schunk zu realisieren ist. Im Teststand kommt das Modell FT-Ome- ga85 mit einem Messbereich von bis zu $950 \mathrm{~N}$ bzw. $40 \mathrm{Nm}$ zum Einsatz (Abbildung 6).

Um die Anwendungsmöglichkeiten des zugrunde liegenden Prinzips zu zeigen, werden diese auf ein reales Einsatzgebiet abgebildet. Ein Beispiel ist die Überwachung des Zustandes verwendeter Werkzeuge, wobei ermittelte Messwerte und Ergebnisse sowie Protokolle direkt im in das Werkzeug eingebrachten RFID-Transponder abgelegt werden. So wird es möglich, vor dem Einsatz des Greifers aktuelle Testergebnisse auszulesen und so nötig Prozessparameter anzupassen, Ersatzteile bereitzuhalten und anschließend die abgelegten Daten durch neuere zu ergänzen. Auf diese Weise können die erreichbaren Genauigkeiten eines Werkzeuges mit den Anforderungen an den Fertigungsprozess abgeglichen werden. Bereits vor der Inbetriebnahme können Abweichungen prognostiziert und über die Freigabe der Bearbeitung entschieden werden. Doch nicht nur an der Maschine, direkt im Lager kann die Vorauswahl erfolgen, sodass auch mit höherem Verschleiß behaftete Werkzeuge noch für Fertigungsaufgaben mit geringeren Qua- litätsansprüchen Verwendung finden können. Wird im Einsatz die voreingestellte Grenze der Werkzeugparameter überschritten und ist eine Kalibrierung nicht mehr möglich, so kann dies automatisiert gemeldet und das Werkzeug für den Einsatz gesperrt werden. Durch die ständige Überwachung liegen die entsprechenden Ersatzteile schon einige Zeit vor dem Ausfall bereit.

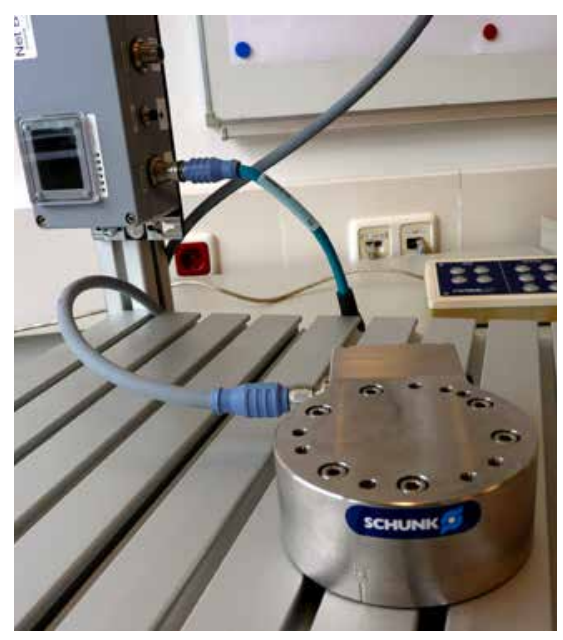

Abb. 6) Foto des Kraftmesssensors FT-Omega85 


\section{FAZIT UND AUSBLICK}

Basierend auf den Untersuchungen zu i4.0-Entitäten ist ein Messstand zur dynamischen Ermittlung von Aktorparametern im industriellen Umfeld entworfen und ein erstes Mock-up erstellt worden. Im weiteren Projektverlauf wird der Teststand aufgebaut, sodass Versuchsdurchführungen und Untersuchungen der Kommunikationswege möglich werden. Auf Basis der gewonnenen Erkenntnisse werden Ansatzpunkte zur intelligenten Vernetzung von Entitäten im produktionstechnischen Umfeld betrachtet. Es wird gezeigt, dass die technischen Voraussetzungen für ein solches System bereits vorhanden sind und lediglich eine Umsetzung erfolgen muss. Weiter werden Möglichkeiten für den Einsatz in verschiedenen Branchen, etwa Life Science oder Kunststoffverarbeitung aufgezeigt. Als Teilprojekt der vierten industriellen Revolution wird so ein Beitrag für den Wirtschaftsstandort Deutschland geleistet. Ziel ist der Wandel bestehender hierarchischer Betriebsstrukturen hin zum cyberphysischen Produktionssystem.

In der Synthese können die im Fallbeispiel verwendeten Strukturen auf ein größeres Anwendungsfeld ausgeweitet werden. So ist es häufig etwa möglich, durch Sensordatenfusion die Aussagefähigkeit von Messungen zu steigern und so das Ersatzteilmanagement oder die Produktionsqualität zu verbessern. Durch die Erweiterung des Teststands um einen Roboter sind deutlich komplexere Strukturen abbildbar, sodass hier eine gute Grundlage für weiterführende Projekte gelegt wird. Auch der Einsatz verschiedener Werkzeuge und neuerer Sensoren ist denkbar.

\section{LITERATUR}

CNC-kompakt (n/a) Kabel 4-adrig geschirmt. http:// www.cnc-kompakt.de/images/product_images/original_images/kabel_geschirmt.jpg. Accessed 15 Sep 2014

Epple U, Bangemann T, Barbian M, Bauer C (2014) Statusreport Industrie 4.0 - Gegenstände, Entitäten, Komponenten. http://www.vdi.de/fileadmin/vdi_de/ redakteur_dateien/gma_dateien/VDI_Industrie_4.0 Komponenten_2014.pdf. Accessed 08 Sep 2014

Kagermann H, Wahlster W, Helbig J (2013) Umsetzungsempfehlungen für das Zukunftsprojekt Industrie 4.0. Abschlussbericht des Arbeitskreises Industrie 4.0. http://www.bmbf.de/pubRD/Umsetzungsempfehlungen_Industrie4_0.pdf. Accessed 08 Sep 2014

Kräußlich W (2013) Sensorik und IO-Link als Wegbereiter für die Fabrik der Zukunft. http://www.konstruktion. de/topstory/sensorik-und-io-link-als-wegbereiter-furdie-fabrik-der-zukunft. Accessed 13 Jun 2014

Lachello L, Wratil P, Meindl A, Schönegger S, Karunakaran BS, Song H, Potier S (2013) Systemvergleich: Die 5 wesentlichen Systeme. Industrial Ethernet Facts $8(2): 1-36$

Meindl A (2014) Ultraschnelle Automatisierung: reACTION Technology. Automotion 2014(5):3-6

Reiff-Stephan J (2013) Probleme mit dem Greifen. A\&D Vorsprung Automation 15(4):116-119

Reiff-Stephan J, Lipinski R von (2014) Selbstoptimierende Prozesse als Schlüsseltechnologien zu Automatisierung der FVK-Produktion. In: Purschwitz A, Hartstock A, Schneider R (eds) Tagungsband zur 11. AALE-Fachkonferenz. Deutscher Industrieverlag, München, pp 379-382

Reinhart G, Engelhardt P, Geiger F, Philipp TR, Wahlster W, Zühlke D, Schlick I Becker T, Löckelt M, Pirvu B, Stephan P, Hodek S, Scholz-Reiter B, Thoben K, Gorldt C, Hribernik KA, Lappe D, Veigt M (2013) Cyber-Physische Produktionssysteme. Produktivitäts- und Flexibilitätssteigerung durch die Vernetzung intelligenter Systeme in der Fabrik. wt Werkstattstechnik online 103(2):84-89

Sauerer J (2013) Smart Sensors. In: Reuter A, Schütze A (eds) Sensorik für Erneuerbare Energien und Energieeffizienz. Beiträge zum Workshop AMA Fachverband für Sensorik und ForschungsVerbund Erneuerbare Energien, März 2013, Berlin, pp 18-24

Spath D (ed) (2013) Produktionsarbeit der Zukunft Industrie 4.0. Studie. Fraunhofer-Verlag, Stuttgart

SPS-MAGAZIN (2011) Ventilinselstecker ersetzen aktorseitig die bisherige Parallelverdrahtung von Ventilinseln durch eine schlanke serielle IO-Link-Verbindung. http://www.sps-magazin.de/bilder/artikel/77879.jpg. Accessed 15 Sep 2014

Vogel-Heuser B, Rösch S, Friedrich A, Göhner P (2013) CPS - eine Fallstudie. Eine Anwendung von CPS im Bereich der Wartung in der Produktautomatisierung. atp edition 55(4):50-56

Zhang L, Fallah YP, Jihene R (2013) Cyber-Physical Systems: Computation, Communication, and Control. Intl ] Distrib Sensor Netw 2013(475818):1 -2. doi: $10.1155 / 2013 / 475818$

\section{AUTOREN}

M. Eng. René von Lipinsk Technische Hochschule Wildau Automatisierungstechnik rene.von_lipinski@th-wildau.de

Prof. Hon.-Prof. Dr.-Ing. Jörg Reiff-Stephan Technische Hochschule Wildau

Professur Automatisierungstechnik/Produktionstechnik joerg.reiff-stephan@th-wildau.de

M. Eng. Markus Richte Technische Hochschule Wildau Automatisierungstechnik markus.richter@th-wildau.de 